\title{
Ethnicity and Multiple Sclerosis Prevalence in East London
}

\author{
Christo Albor, Tim du Sautoy, Narmadha Kali Vanan, Kambiz Boomla, and Klaus Schmierer
}

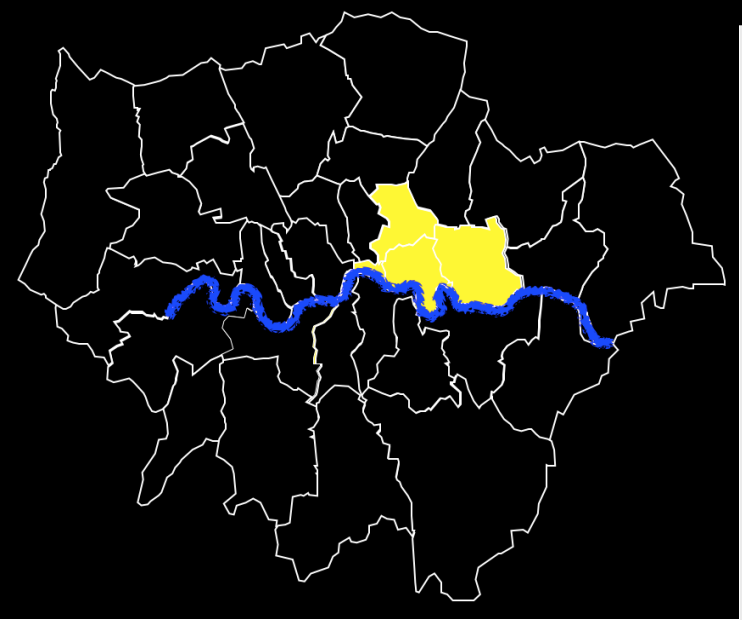

BACKGROU N D

Establishing the prevalence in the UK of multiple sclerosis (MS) among ethnic minorities hailing from low prevalence countries is of interest to explore factors involved in MS causation. East London is home to an ethnically diverse population, thus providing an excellent backdrop to update previously reported prevalence data [1]. Real-time prevalence data derived from primary care in East London were used.

\section{METHODS}

We studied the population residing in the four London boroughs closest to The Royal London Hospital (Barts Health NHS Trust), where our MS services are based. These boroughs, highlighted in yellow above, are Tower Hamlets, Newham, Hackney, and the City. For simplicity, we refer to these boroughs together as 'East London'.

Electronic records from GPs in these boroughs were queried from a 2014 extract of a primary care records database provided by the Clinical

Effectiveness Group at the Centre for Primary Care (Blizard Institute). From this we derived the number of patients in East London with a diagnosis of MS, grouped by ethnicity and into 5 -year age brackets. We derived the total number of people registered with GPs in East London in the same way. We then calculated the age-standardised MS prevalence, for the total population, and separately for White, Black and South Asian populations. Age-standardisation followed the direct method based on the 2013 European Standard Population.

\section{RE S U L T S}

A total of 907,151 patients were registered with GP practices in East London. Of these, $776 \mathrm{had}$ a diagnosis of MS. The table below shows a breakdown of this MS population by sex and ethnicity.

Overall MS prevalence was $111 / 100,000$ (152 for women, 70 for men). Prevalence was 180, 74, and 29 for the White, Black and South Asian populations, respectively. The female:male ratio was 2.2:1, 2.1:1 and 2.8:1, respectively. The bar-chart illustrates the sex and ethnic-specific prevalence rates.

\section{No. people with MS in East London}

\begin{tabular}{r|ccc} 
& Female & Male & Total (\%) \\
\hline White & 345 & 162 & $507(65)$ \\
Black & 77 & 34 & $111(14)$ \\
South Asian & 47 & 24 & $71(9)$ \\
Other & 15 & 9 & $24(3)$ \\
Unknown & 38 & 25 & $63(8)$ \\
\hline Total & $\mathbf{5 2 2}$ & $\mathbf{2 5 4}$ & $\mathbf{7 7 6}$
\end{tabular}

\section{I S C U S S I O N}

Compared to the White population MS prevalence remains considerably lower amongst Black and South Asian people, by $59 \%$ and $84 \%$, respectively. However, compared to available data in Africa and South Asia, MS is up to 10 times (or more) prevalent among Blacks and South Asians living in the UK than their territorial ancestry [3].

Whilst this difference may partly be explained by under-diagnosis and under-reporting the magnitude of the increase in risk suggests an important role of an environmental agent (or several thereof). As we were not (yet) in the position to separate minorities born in the UK from those born abroad, no definitive conclusions can be drawn at this stage.

Despite general agreement of our estimates with previous studies in the UK, we detected an important difference: whilst previously Black people were found to have the lowest MS risk [1], in our study their risk was second only to White people. The rise of MS among Black people warrants further research, though in the UK Black people are far from 'overtaking' the White population as has been reported in two studies from the US [2].

\section{Sex and Ethnic-specific MS Prevalence (per 100K)}

300

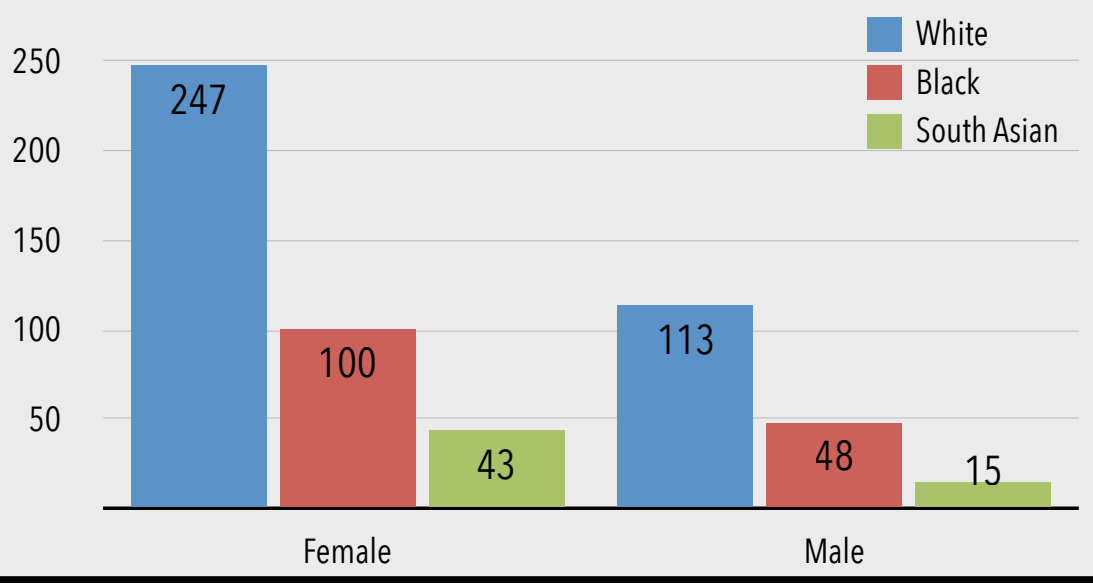

\section{REFERENCES}

[1] Dean et. al. Br Med J. 1976;1:861-864; Dean et al. Br J Prev Soc Med. 1977;31:141-147.

[2] Langer-Gould et al. Neurology. 2013;80:1734-1739; Wallin et al. Brain. 2012;135(Pt 6):1778-85 [3] The Atlas of MS 2013, www.msif.org. 